IPMU 10-0047

\title{
BLACK HOLES CONSTITUTE ALL DARK MATTER
}

\author{
Paul H. Frampton* \\ Department of Physics and Astronomy, University of North Carolina, Chapel Hill, USA and \\ Institute for the Mathematics and Physics of the Universe, University of Tokyo, Japan. \\ *E-mail: frampton@physics.unc.edu and paul.frampton@ipmu.jp
}

The dimensionless entropy, $\mathcal{S} \equiv S / k$, of the visible universe, taken as a sphere of radius 50 billion light years with the Earth at its "center", is discussed. An upper limit $\left(10^{112}\right)$, and a lower limit $\left(10^{102}\right)$, for $\mathcal{S}$ are introduced. It is suggested that intermediate-mass black holes (IMBHs) constitute all dark matter, and that they dominate $\mathcal{S}$.

Keywords: Dark matter, black hole, entropy, halo.

\section{Introduction}

Two references useful for further information about the material of this talk are:

(1) P.H.F. and T.W. Kephart. Upper and Lower Bounds on Gravitational Entropy. JCAP 06:008 (2008) and

(2) P.H.F. Identification of All Dark Matter as Black Holes. arXiv:0905.3632 [hep-th]. JCAP 0910:016 (2009).

\section{The Entropy of the Universe.}

As interest grows in pursuing alternatives to the Big Bang, including cyclic cosmologies, it becomes more pertinent to address the difficult question of what is the present entropy of the universe?

Entropy is particularly relevant to cyclicity because it does not naturally cycle but has the propensity only to increase monotonically. In one recent proposal, the entropy is jettisoned at turnaround. In any case, for cyclicity to be possible there must be a gigantic reduction in entropy (presumably without violation of the second law of thermodynamics) of the visible universe at some time during each cycle.

Standard treatises on cosmology address the question of the entropy of the universe and arrive at a generic formula for a thermalized gas of the form

$$
S=\frac{2 \pi^{2}}{45} g_{*} V_{U} T^{3}
$$


where $g_{*}$ is the number of degrees of freedom, $T$ is the Kelvin temperature and $V_{U}$ is the volume of the visible universe. From Eq.(1) with $T_{\gamma}=2.7^{0} \mathrm{~K}$ and $T_{\nu}=$ $T_{\gamma}(4 / 11)^{1 / 3}=1.9^{0} \mathrm{~K}$ we find the entropy in CMB photons and neutrinos are roughly equal today

$$
S_{\gamma}\left(t_{0}\right) \sim S_{\nu}\left(t_{0}\right) \sim 10^{88}
$$

Our topic here is the gravitational entropy, $S_{\text {grav }}\left(t_{0}\right)$. Following the same path as in Eqs. (112) we obtain for a thermal gas of gravitons $T_{\text {grav }}=0.91^{\circ} \mathrm{K}$ and then

$$
S_{\text {grav }}^{(\text {thermal })}\left(t_{0}\right) \sim 10^{86}
$$

This graviton gas entropy is a couple of orders of magnitude below that for photons and neutrinos. This graviton gas entropy is a couple of orders of magnitude below that for photons and neutrinos. But there are larger contributions to gravitational entropy from elsewhere!!!

\section{Upper Limit on the Gravitational Entropy.}

We shall assume that dark energy has zero entropy and we therefore concentrate on the gravitational entropy associated with dark matter. The dark matter is clumped into halos with typical mass $M($ halo $) \simeq 10^{11} M_{\odot}$ where $M_{\odot} \simeq 10^{57} \mathrm{GeV} \simeq 10^{30} \mathrm{~kg}$ is the solar mass and radius $R($ halo $)=10^{5} \mathrm{pc} \simeq 3 \times 10^{18} \mathrm{~km} \simeq 10^{18} r_{S}\left(M_{\odot}\right)$. There are, say, $10^{12}$ halos in the visible universe whose total mass is $\simeq 10^{23} M_{\odot}$ and -corresponding Schwarzschild radius is $r_{S}\left(10^{23} M_{\odot}\right) \simeq 3 \times 10^{23} \mathrm{~km} \simeq 10 \mathrm{Gpc}$. This happens to be the radius of the visible universe corresponding to the critical density. This has led to an upper limit for the gravitational entropy is for one black hole with mass $M_{U}=10^{23} M_{\odot}$.

Using $S_{B H}\left(\eta M_{\odot}\right) \simeq 10^{77} \eta^{2}$ corresponds to the holographic principle for the upper limit on the gravitational entropy of the visible universe:

$$
S_{\text {grav }}\left(t_{0}\right) \leq S_{\text {grav }}^{(\mathrm{HOLO})}\left(t_{0}\right) \simeq 10^{123}
$$

which is 37 orders of magnitude greater than for the thermalized graviton gas in Eq.(3) and leads us to suspect (correctly) that Eq.(3) is a gross underestimate. Nevertheless, Eq. (4) does provide a credible upper limit, an overestimate yet to be refined downwards below, on the quantity of interest, $S_{\text {grav }}\left(t_{0}\right)$.

The reason why a thermalized gas of gravitons grossly underestimates the gravitational entropy is because of the 'clumping' effect on entropy. Because gravity 
is universally attractive its entropy is increased by clumping. This is somewhat counter-intuitive since the opposite is true for the familiar 'ideal gas'. It is best illustrated by the fact that a black hole always has 'maximal' entropy by virtue of the holographic principle.

\section{Lower Limit on Gravitational Entropy}

It is widely believed that most, if not all, galaxies contain at their core a supermassive black hole with mass in the range $10^{5} M_{\odot}$ to $10^{9} M_{\odot}$ with an average mass about $10^{7} m_{\odot}$. Each of these carries an entropy $S_{B H}$ (supermassive) $\simeq 10^{91}$. Since there are $10^{12}$ halos this provides the lower limit on the gravitational entropy of

$$
S_{\text {grav }}\left(t_{0}\right) \geq 10^{103}
$$

which, by now. provides an eight order of magnitude window for $S_{\text {grav }}\left(t_{0}\right)$.

The lower limit in Eq.(5) from the galactic supermassive black holes may be largest contributor to the entropy of the present universe but this seems to us highly unlikely because they are so very small. Each supermassive black hole is about the size of our solar system or smaller and it is intuitively unlikely that essentially all of the entropy is so concentrated.

Gravitational entropy is associated with the clumping of matter because of the long range unscreened nature of the gravitational force. This is why we propose that the majority of the entropy is associated with the largest clumps of matter: the dark matter halos associated with galaxies and cluster.

\section{Intermediate Mass Black Holes}

If we consider normal baryonic matter, other than black holes, contributions to the entropy are far smaller. The background radiation and relic neutrinos each provide $\sim 10^{88}$. We have learned in the last decade about the dark side of the universe. WMAP suggests that the pie slices for the overall energy are $4 \%$ baryonic matter, $24 \%$ dark matter and $72 \%$ dark energy. Dark energy has no known microstructure, and especially if it is characterized only by a cosmological constant, may be assumed to have zero entropy. As already mentioned, the baryonic matter other than the SMBHs contributes far less than $\left(S_{U}\right)^{\text {min }}$.

This leaves the dark matter which is concentrated in halos of galaxies and clusters.

It is counter to the second law of thermodynamics when higher entropy states are available that essentially all the entropy of the universe is concentrated in SMBHs. 
The Schwarzschild radius for a $10^{7} M_{\odot}$ SMBH is $\sim 3 \times 10^{7} \mathrm{~km}$ and so $10^{12}$ of them occupy only $\sim 10^{-36}$ of the volume of the visible universe.

Several years ago important work by $\mathrm{Xu}$ and Ostriker showed by numerical simulations that IMBHs with masses above $10^{6} M_{\odot}$ would have the property of disrupting the dynamics of a galactic halo leading to runaway spiral into the center. This provides an upper limit $\left(M_{I M B H}\right)^{\max } \sim 10^{6} M_{\odot}$.

Gravitational lensing observations are amongst the most useful for determining the mass distributions of dark matter. Weak lensing by, for example, the HST shows the strong distortion of radiation from more distant galaxies by the mass of the dark matter and leads to astonishing three-dimensional maps of the dark matter trapped within clusters. At the scales we consider $\sim 3 \times 10^{7} \mathrm{~km}$, however, weak lensing has no realistic possibility of detecting IMBHs in the forseeable future.

Gravitational microlensing presents a much more optimistic possibility. This technique which exploits the amplification of a distant source was first emphasized in modern times (Einstein considered it in 1912 unpublished work) by Paczynski. Subsequent observations found many examples of MACHOs, yet insufficient to account for all of the halo by an order of magnitude. These MACHO searches looked for masses in the range $10^{-6} M_{\odot} \leq M \leq 10^{2} M_{\odot}$.

The time $t_{0}$ of a microlensing event is given by

$$
t_{0} \equiv \frac{r_{E}}{v}
$$

where $r_{E}$ is the Einstein radius and $v$ is the lens velocity usually taken as $v=$ $200 \mathrm{~km} / \mathrm{s}$. The radius $r_{E}$ is proportional to the square root of the lens mass and numerically one finds

$$
t_{0} \simeq 0.2 y\left(\frac{M}{M_{\odot}}\right)^{1 / 2}
$$

so that, for the MACHO masses considered, $2 h \leq t_{0} \leq 2 y$.

\section{Cosmological Entropy Considerations.}

The cosmological entropy range

$$
102 \leq \log _{10} S_{U} \leq 112
$$


is the first of two interesting windows which are the subject. Conventional wisdom is $S_{U} \sim\left(S_{U}\right)^{\min }=10^{102}$.

\section{Intermediate Mass Black Holes and Microlensing Longevity}

\begin{tabular}{||c|c|c|c||c||}
\hline \hline $\log _{10} n_{\max }$ & $\log _{10} \eta$ & $\log _{10} S_{\text {halo }}$ & $\log _{10} S_{U}$ & $t_{0}$ (years) \\
\hline \hline 8 & 2 & 88 & 100 & 2 \\
\hline 7 & 3 & 89 & 101 & 6 \\
\hline 6 & 4 & 90 & 102 & 20 \\
\hline 5 & 5 & 91 & 103 & 60 \\
\hline 4 & 6 & 92 & 104 & 200 \\
\hline \hline
\end{tabular}

(Assumes $\rho_{I M B H} \sim 1 \% \rho_{D M}$ )

\section{Observation of IMBHs}

Since microlensing observations already impinge on the lower end of the range (??) and the Table, it is likely that observations which look at longer time periods, have higher statistics or sensitivity to the period of maximum amplification can detect heavier mass IMBHs in the halo. If this can be achieved, and it seems a worthwhile enterprise, then the known entropy of the universe could be increased by more than two orders of magnitude.

There exists interesting other analyses pertinent to existence of massive halo objects:

J. Yoo, J, Chanamé and A. Gould, Astrophys. J. 601, 311 (2004). astro-ph/0307437.

It is this entropy argument based on holography and the second law of thermodynamics which is the most compelling supportive argument for IMBHs. If each galaxy halo asymptotes to a black hole the final entropy of the universe will be $\sim 10^{112}$ as in Eq.(8) and the universe will contain just $\sim 10^{12}$ supergigantic black holes. Conventional wisdom is that the present entropy due entirely to SMBHs is only $\sim 10^{-10}$ of this asymptopic value. IMBHs can increase the fraction up to $\sim 10^{-8}$, closer to asymptopia and therefore more probable according to the second law of thermodynamics.

There are several previous arguments about the existence of IMBHs and they have put upper limits on their fraction of the halo mass. The entropy arguments are new and provide additional motivation to tighten these upper bounds or discover the 
halo black holes. One observational method is high longevity microlensing events. It is up to the ingenuity of observers to identify other, possibly more fruitful, methods some of which have already been explored in a preliminary way.

\section{Post-conference update}

Since the SCGT09 conference took place, the paper:

Paul H. Frampton, Masihiro Kawasaki, Fuminobu Takahashi and Tsutomu Yanagida

IPMU-09-0157 (December 2009).

Primordial Black Holes as All Dark Matter

arXiv:1001.2308 [hep-ph].

shows that it is possible to form black holes in the early universe with mass $10^{5} M_{\odot}$ and with sufficient abundance to provide all of the dark matter.

\section{Acknowledgements}

This work was supported in part by the World Premier International Research Center Initiative (WPI initiative), MEXT, Japan and by U.S. Department of Energy Grant No. DE-FG02-05ER41418. 